\title{
Philonsorbonne
}

11 | 2017

Année 2016-2017

\section{Le mythe du dehors. Sartre face à la philosophie digestive}

Alexandre COUTURE-MINGHERAS

\section{OpenEdition}

Journals

Édition électronique

URL : https://journals.openedition.org/philonsorbonne/842

DOI : $10.4000 /$ philonsorbonne.842

ISSN : $2270-7336$

Éditeur

Publications de la Sorbonne

Édition imprimée

Date de publication : 1 janvier 2017

Pagination : $9-30$

ISSN : 1255-183X

\section{Référence électronique}

Alexandre COUTURE-MINGHERAS, « Le mythe du dehors. Sartre face à la philosophie digestive »,

Philonsorbonne [En ligne], 11 | 2017, mis en ligne le 05 janvier 2017, consulté le 08 juin 2021. URL:

http://journals.openedition.org/philonsorbonne/842; DOI : https://doi.org/10.4000/philonsorbonne. 842

(c) Tous droits réservés 


\title{
Le mythe du dehors. Sartre face à la philosophie digestive
}

\author{
Alexandre Couture-Mingheras
}

\section{Introduction}

L'expression de « philosophie digestive $»^{1}$, mentionnée pour la première fois dans un célèbre article de Jean-Paul Sartre de 1939, "Une idée fondamentale de la phénoménologie de Husserl : l'intentionnalité », et dans la Transcendance de l'ego de 1936, ne constitue pas à proprement parler un concept clair et délimité. Sartre se montre allusif, visant, sans plus de précision, le néo-kantisme, le psychologisme et l'empiriocriticisme. Mais, en dépit du peu d'importance qui lui est quantitativement accordée, il s'agit, pour reprendre la distinction d'Eugen Fink, d'une catégorie non pas thématique (ce que pense l'auteur) mais opératoire (à l'aide de laquelle le philosophe pense mais qui reste en retrait de la thématisation qu'elle sert), qui est absolument centrale, traversant l'œuvre de Sartre jusqu'à L'Etre et le Néant en 1943. Ce flou qui entoure le syntagme et la relative légèreté avec

\footnotetext{
1. Ou « alimentaire », cf. J.-P. Sartre, La Transcendance de l'ego. Esquisse d'une description phénoménologique, introduction, notes et appendices par S. Le Bon, Paris, Vrin, 2012, p. 109. Nous nous référons à cette édition pour les deux textes. Nous renvoyons cela dit à celle de Vincent de Coorebyter, qui date de 2003. Elle offre une introduction stimulante qui éclaire notamment l'histoire des rapports de Sartre à l'épistémologie d'Ernst Mach et d'Emile Meyerson, mais aussi au courant "idéaliste» français d'André Lalande et de Léon Brunschvicg, auprès desquels il s'est formé. Il s'agit moins ici de montrer la manière dont Sartre récupère (en les critiquant) ces auteurs que d'éclairer son positionnement philosophique et l'élaboration de son concept d'intentionnalité contre une "philosophie digestive » qui, telle qu'elle est mentionnée dans les textes, a une valeur paradigmatique (comme philosophie de la « représentation » qui fait fond sur la notion « d'intériorité »).
} 
laquelle Sartre rejette sans autre forme de procès des «traditions » (ce qu'atteste le «-isme » généralisant) qu'il ne prend point la peine de discuter et de nuancer (qu'est-ce que le « néo-kantisme » en général ?), relèvent sans doute d'un choix significatif. Cette imprécision flagrante, de l'ordre de la provocation, tient déjà au style philosophique de Sartre, lequel recourt habituellement aux auteurs pour se dessiner une place dans l'espace de la pensée et non pour en restituer fidèlement le texte. Mais une autre raison s'ajoute à cela, qui tient à la valeur paradigmatique dévolue à la "philosophie digestive », laquelle désigne le régime de la philosophie représentationaliste qui interroge la fidélité de la perception à son objet, en accord avec la définition scolastique de la vérité comme adaequatio rei et intellectus, régime avec lequel Sartre s'efforce de rompre : en effet, cela revient à construire le problème d'une telle façon qu'il en devient insoluble, puisqu'il parait impossible de sortir de l'image (ou percept) pour la comparer à son dehors si ce n'est à recourir à un deus ex machina capable d'assurer l'adéquation entre sujet et objet et dont le Dieu cartésien dans la quatrième des Méditations métaphysiques est un exemple emblématique.

Sartre, alors boursier à l'Institut de Berlin en 1933-1934, trouve en la phénoménologie, alors quasiment inconnue en France (et dont Lévinas par sa thèse sur La théorie de l'intuition chez Husserl, publiée en 1930, fut le premier introducteur) un allié de taille contre "l'idéalisme» triomphant dans l'université française dans les années 1920, avec au premier chef Léon Brunschvicg. Sartre, on le sait, reconnait à la phénoménologie, comme «méthode descriptive», le mérite de proposer une manière autre de faire de la philosophie, où la pensée se met au service d'un réel sur lequel se calibrer plutôt que de soumettre ce dernier à l'aune de ses catégories : le logos prend en charge la description du phanestai, et cette récupération singulière, qui dissocie la phénoménologie de sa problématique originellement épistémologique (celle d'une " science rigoureuse »), conduit à un « réalisme phénoménologique ». La phénoménologie lui paraît en effet offrir, avec son concept central d'intentionnalité, un argument de poids contre toute forme d'idéalisme où le sujet sonde ses images pour s'interroger par suite, dans une démarche que l'auteur juge vouée à l'échec, sur ce qui atteste ou non de leur lien au réel ${ }^{2}$. À condition toutefois que le vécu décrit n'ait plus rien de subjectif, sans quoi le descriptivisme pur (affranchi des catégories métaphysiques) qui, de façon minimale, est définitoire de la phénoménologie, retomberait dans une forme d'introspectionnisme.

Cette exigence descriptiviste engage ainsi chez Sartre une externalisation $\mathrm{du}$ vécu, i.e. une manière de cesser d'enrégimenter l'expérience dans la grammaire subjectiviste, c'est-à-dire de la concevoir comme ce qui provient de l'extérieur pour entrer ensuite dans la sphère de la subjectivité. Or, en partant de la déconstruction de ce que l'on peut appeler le «mythe de l'intériorité », nous voudrions tirer une conclusion quant à

2. A. Guigot, Sartre. Liberté et histoire, Paris, Vrin, 2007, p. 13 : « La phénoménologie invite à un recommencement en soi-même de l'aventure du vrai ». 
l'attitude philosophique qui, de manière plus générale, consiste à vouloir tout mettre au dehors, s'appuyant sur une structure dualiste (de quoi serait le dehors si ce n'est celui d'un dedans ?) à laquelle elle emprunte son lexique pour affirmer immédiatement après que ce dualisme n'est que d'apparence et qu'il n'y a jamais eu qu'un seul terme, le dehors.

Il s'agira donc, après avoir rendu compte de la refonte sartrienne du concept d'intentionnalité, de tirer un enseignement général (pour le temps présent) quant à la posture philosophique qui, voulant échapper à ce qui est devenu aujourd'hui un lieu commun de la critique, le dit «mythe» de l'intériorité, s'avère à nos yeux intenable. Si le «dedans » pose un problème au sein de la théorie plus globale de la connaissance, nous ne croyons pas, pour notre part, que cette «course » à l'externalisation suffise à tout résoudre ni qu'elle se fasse sans prix à payer.

\section{Une lecture » réaliste »de l'intentionnalité}

Mais revenons au préalable sur la métaphore filée de la « digestion » dans le texte de 1939, qui, loin de se réduire à un simple trope, relève au contraire de la stratégie que met en place Sartre et à l'aune de laquelle la défense du « dehors » tire sa justification : la radicalité du dehors constitue le symétrique inverse de la radicalité du dedans. Mais il en va également d'une rhétorique qui sert la thèse de l'auteur, car, et cela est significatif, le texte est d'emblée axiologique (il serait à cet endroit pour le moins difficile de trouver quelque chose comme une argumentation en bonne et due forme, i.e. sous la forme d'un texte qui se voudrait purement théorique), mettant en balance d'un côté une extériorité joyeuse et riche, faite de villes, de chemins de campagnes et d'hommes, et, de l'autre, une intériorité écœurante, dont rendent compte les images de «moite intimité» et de «bave». Qu'il s'agisse là d'un texte de combat, et non véritablement d'une introduction, de l'ordre de l'exégèse, de la phénoménologie husserlienne en France, c'est ce que confirme cette note tirée des Carnets de la drôle de guerre où Sartre déclare sans ambages que son adhésion à un certain type de réalisme, au premier sens du terme, comme ce qui soutient l'idée qu'il existe une réalité indépendante du sujet et de ses catégories, procède avant tout d'un parti pris moral. «Le réalisme c'était aussi l'affirmation de la résistance du monde et de ses dangers contre la philosophie dissolvante de l'idéalisme, l'affirmation du Mal contre la philosophie optimiste de l'unification. Mais il avait, j'imagine, une autre source : il venait de mon émerveillement devant le monde et l'époque que je découvrais. Comment admettre que tant de charmes, tant de plaisirs à conquérir et tant de beaux dangers étaient seulement des ombres, des "représentations" mal unifiées (...). J'étais réaliste par morale $\iota^{3}$.

3. J.-P. Sartre, Carnets de la drôle de guerre, Paris, Gallimard, 1983, p. 280-286. 
À l'inverse, la philosophie digestive a le tort, selon Sartre, de nous faire perdre le réel, concevant la perception comme un processus « d'assimilation » et de " nutrition ». Tout objet, du fait même d'être perçu, perd la qualité qui en était définitoire, son extériorité, et devient un contenu pour la conscience : le sujet désespère d'accéder au réel, et, croyant palper quelque chose d'extérieur, ne fait au fond que jouer avec ses propres images. Si Sartre juge le biffage du réel inévitable dans le cas d'un tel immanentisme (ou «philosophie douillette de l'immanence»), c'est donc parce que ce dernier, partant de la conscience, se trouve incapable de la dépasser, que s'étant donné pour point de départ la subjectivité, il ne saurait en sortir. Il est, pour le dire de deux mots, toujours sur le départ, la chose "même" lui étant, une fois donnée, soustraite comme telle et transformée en "sa » chose. Or si jamais d'une image ne sortira le réel, pas plus que de l'intériorité la transcendance, c'est donc qu'il est absurde de tenter de réunir artificiellement, pour ainsi dire de l'intérieur, deux pôles dont l'un est radicalement extérieur, et qu'il faut, selon Sartre, cesser de partir du sujet, point de départ qui, en effet, engage toute une problématique de l'accès au réel - qui, du reste, suppose que l'on ne soit pas au réel, tant il est vrai qu'on n'accède à quelque chose qu'en n'y étant pas. Aussi la déconstruction du problème de l'accès a-t-elle partie liée avec la destruction de tout ce qui met le réel à distance, à savoir l'intériorité, qui sépare, par toute l'épaisseur de ses affects et de ses représentations, le sujet du monde. Si la « digestion », ou l'internalisation de l'extériorité, se révèle en dernière instance adossée à une conception substantialiste de la conscience (c'est parce qu'elle est une res que la conscience peut contaminer l'extériorité et se l'approprier, que, pour reprendre l'exemple sartrien, elle peut faire entrer "l'arbre» dans ses «estomacs sombres » ${ }^{4}$ ), alors Sartre doit, pour redonner ses lettres de noblesse au réel, se battre sur deux fronts : d'une part rompre avec le régime représentationaliste qui pense la perception en termes d'inclusion, d'autre part dissocier la conscience de la subjectivité, le sujet de la positivité qui échoit quant à elle aux objets. Contre cette conscience assimilatrice, qui emprisonne dans le circuit de son ipséité tout ce qui prétend y échapper, Sartre sauve le dehors par l'abolition radicale du dedans.

Cette pensée du dehors, qui n'est la contrepartie d'aucun dedans, Sartre la met ainsi en œuvre dans ce court texte de 1939, en recourant de façon stratégique au concept central dans la phénoménologie husserlienne d'intentionnalité. Il faut néanmoins relever que ce concept emprunté à la tradition scolastique, avait chez Franz Brentano, dans sa Psychologie vom empirischen Standpunkt (1874), pour objectif affiché de distinguer le psychique du physique et qu'il reposait, comme le remarque Jean-François Courtine dans La cause de la phénoménologie $e^{5}$, sur le dualisme cartésien entre res cogitans et res extensa. Par la suite, l'intentionnalité, dans la reprise qu'en fait Kazimierz Twardowski dans son opuscule Zur Lehre vom Inhalt

4. J.-P. Sartre, La Transcendance de l'ego, op. cit., p. 111.

5. J.-F. Courtine, La Cause de la phénoménologie, Paris, PUF, « Epiméthée », 2007, p. 21. 
und Gegenstand der Vorstellungen (1894), servait à régler son sort à la question, épineuse dans le second quart du XIX ${ }^{\text {ème }}$ siècle, relative au statut des entités négatives et fictives, et s'arrimait, sous cet angle, à la dichotomie entre contenu (psychique) et objet (physique), au sens où je peux viser un objet sans pour autant que ce dernier corresponde à quoi que ce soit d'existant à l'extérieur. La notion de contenu ${ }^{6}$ suffisait de ce point de vue à conserver le format intentionnaliste de la conscience, mais restait, comme telle, tributaire d'une certaine philosophie de la représentation, le contenu étant à la fois ce que vise la conscience et ce qui lui sert d'intermédiaire avec l'objet, ce qui se pense en termes d'inclusion réelle. Inutile de dire que le texte sartrien contraste de façon saisissante avec l'inintentionnalité brentanienne et la philosophie de l'Inhalt («Qu'est-ce qu'une table, un rocher, une maison? Un certain assemblage de "contenus de conscience", un ordre de ces contenus. Ô philosophie alimentaire! $\rangle^{7}$ ). Le fait que Sartre passe sous silence cette histoire du concept d'intentionnalité n'a à cet égard, semble-t-il, rien d'anodin : se délester de cette histoire le conduit à proposer, refusant d'entrer sur le terrain de la glose, une intentionnalité d'emblée réaliste - qui refuse de se justifier, i.e. d'être acquise en délestant au maximum le concept de la part idéaliste dont il est historiquement porteur.

Si l'on peut donc à bon droit parler de radicalisation de l'intentionnalité, c'est au sens où, passant outre l'histoire même de son concept (le dissociant de tout projet de fondation d'une "psychologie scientifique»), Sartre soutient que la conscience s'épuise totalement dans ce qu'elle vise et se trouve tout entière prise dans la transitivité de ce « de » (conscience « de »), sans possibilité pour elle de refluer : se trouve ici raturé tout lieu à partir duquel le sujet pourrait se retirer et se recueillir. Mais ce serait encore trop peu dire que d'affirmer qu'elle se rapporte au monde : elle est ce rapport. Le coup de maître de Sartre consiste en effet à détourner la grammaire du rapport, qui est celle d'un rapport entre deux positivités distinctes, au profit d'un rapport reconçu comme ouverture. "Ce n'est pas dans je ne sais quelle retraite que nous nous découvrirons : c'est sur la route, dans la ville, au milieu de la foule, chose parmi les choses, homme parmi les hommes $»^{8}$. Il est donc tout à fait licite de qualifier cette lecture de l'intentionnalité de "réaliste », dans la mesure où le rapport qu'est la conscience ne peut s'effectuer qu'en se prélevant sur un déjà-là, sur un réel qui, loin d'être

6. Cf. J. Benoist, Entre acte et sens. La théorie phénoménologique de la signification, Paris, Vrin, 2002, p. 19: "Twardowski n'est toutefois pas entièrement clair sur le statut de cette notion de "contenu". S'il prend bien soin de la distinguer de la représentation elle-même en tant qu'effectivité psychique, et paraît l'entendre en un sens quasi-sémantique, il semble tout de même la rattacher en quelque mesure à cette effectivité, dans le souci de lui donner un "site" ontologique. Par là même, il s'expose à la critique peu charitable de Husserl, qui l'accuse de confondre deux sens de l'immanence : celle, purement idéale, du "sens" à l'acte, et celle d'une inclusion réelle, effective, qui est celle du contenu psychique dans la conscience $»$.

7. J.-P. Sartre, La Transcendance de l'ego, op .cit., p. 109.

8. Ibid., p. 113. 
constitué par le rapport (ce qui serait l'idéalisme pour Sartre), le précède. Mais cette thèse constitutive de tout réalisme minimal, d'une indépendance du monde par rapport à la conscience, ne peut plus ici se dire dans le langage du dualisme, au sens où l'indépendance du réel par rapport à la conscience suppose qu'il y ait quelque chose comme une conscience positive, ce que Sartre, déjà avant l'Être et le Néant, refuse. Si la conscience était grevée d'un reste de positivité, si elle recelait encore en elle le moindre soupçon de res cogitans, c'en serait fini de la pensée du dehors. En d'autres termes, il est important de souligner que le passage de la logique de l'avoir, qui est celle de la digestion, à la logique de la relation, ne suffit pas à entériner la première, si l'on s'entête à penser la relation en termes de rapport entre deux termes. Une pensée du dehors qui pêcherait par manque de radicalité se renverserait en son contraire. Si la conscience n'est pas encore un rien, comme ce sera le cas en 1943, et si elle ne souffre pas dans l'article de 1939 de carence (si elle ne se vit pas comme manque), elle n'est pas pour autant quelque chose. C'est ainsi que l'on doit prendre la mesure du contraste entre d'une part le modèle de clarté dont relèvent les images de légèreté et de translucidité qu'emploie Sartre pour désigner avec lyrisme la conscience (elle qui est « claire comme un grand vent», incapable d'en rester à un «àchez-soi », saisie qu'elle est par un " tourbillon » qui la rejette au-dehors, « près de l'arbre, en pleine poussière ${ }^{9}$ ), et d'autre part le modèle de l'obscurité auquel ressortissent les images par lesquelles Sartre désigne la philosophie digestive (celles de déglutition, de "brouillard mou», de dilution, «d'intimité gastrique $»^{10},\left\langle\right.$ d'estomacs sombres $\left.»^{11}\right)$. La conscience n'étant « rien », plus rien ne fait obstacle entre « elle» (d'un « elle» sans épaisseur) et le monde.

\section{L'externalisation de l'affect et la mort de l'intériorité}

Encore faut-il que le rapport au monde ne se subordonne pas au rapport à soi. Dans la partie finale de l'article de 1939, Sartre défend une thèse qui de prime abord semble contre-intuitive : l'affect ne désigne-t-il pas en effet cette part inéliminable de subjectivité ? La joie n'est-elle pas ce qui m'est le plus propre? Jusqu'ici, en effet, il était question de perception comme perception d'objet, ce qui était rendu possible par le fait que le monde se trouvait dans un rapport d'antériorité « logique » par rapport à la conscience. En d'autres termes, l'objet était objet avant que d'être perçu, ce qui en garantissait la radicale indépendance. Or s'il est nécessaire de reconcevoir la nature de l'affect, c'est bien parce qu'en lui résiste une partie résiduelle d'intériorité et que l'accepter conduirait Sartre à prendre d'une main à la

9. Ibid., p. 111.

10. Ibid., p. 110.

11. Ibid., p. 111. 
conscience «perceptive» ce qu'il donne de l'autre à la conscience «affective»: l'intériorité serait reportée du percept sur l'affect. Cela reviendrait en effet à trop concéder à l'immanentisme : si le dedans triomphe à peu de frais, la pensée du dehors, quant à elle, doit être dépensière, car à manquer de radicalité la moindre assomption d'un "contenu» affectif de conscience suffirait à la renverser. De toute évidence, ce qui est ainsi en ligne de mire, c'est l'idée que la perception recèle une dimension affective et que le monde varie en fonction de nos états d'âme : en un mot, l'idée que le réel est vu à travers nos affects. Ce qui matérialise un tel point de vue introspectionniste, c'est toute cette littérature du récit de soi et de l'autoanalyse, que cristallise la formule-choc qui est sur le point de clore le texte : « Nous voilà délivrés de Proust » ${ }^{12}$.

Mais il est une autre référence majeure, que Sartre passe sous silence ${ }^{13}$ eu égard à l'usage stratégique qu'il en fait pour défendre sa déconstruction du mythe de l'intériorité, à savoir Husserl. En effet, Brentano, dans le célèbre $\S 3$ de sa Psychologie vom empirischen Standpunkt, établit un rapport de fondation entre la représentation (qui est toujours représentation d'objet) et l'affect, soutenant que rien «ne peut être jugé, mais rien non plus ne peut être désiré, rien ne peut être espéré ou craint, qui n'ait d'abord été représenté ${ }^{14}$. La conscience se définit par l'intentionnalité (par le fait qu'elle est conscience $d e$ ), autrement dit elle se caractérise par le fait qu'elle vise un objet. De ce point de vue, l'affect s'avère génétiquement secondaire et se greffe sur la représentation «chosale». Mais, secondarisant l'affect, Brentano est alors conduit à dés-intentionnaliser l'affect: s'il ne peut y avoir de crainte sans représentation de l'objet qui en est la cause, de crainte sans quelque chose à craindre, bref s'il ne peut y avoir d'affect sans intentionnalité, c'est donc que l'affect dépend de la visée mais n'est pas en lui-même intentionnel. On doit alors en conclure, dans cette perspective, que l'affect s'ajoute à la représentation objectale sans pour autant jouir lui-même du même statut, et que l'affect est ce qui résiste en un certain sens au projet brentanien qui consiste à établir une psychologie scientifique. Car si la psychologie possède désormais un domaine qui lui est propre et que la conscience se définit par son intentionnalité, alors que faire de ces affects?

Toujours est-il que Husserl s'inscrit dans cette tradition et reconduit cette forme de duplicité entre l'objet et le non-objet: accorder le primat aux actes objectivants (aux «représentations» en termes brentaniens), qu'est-ce d'autre qu'affirmer par la même occasion que ce qui ne bénéficie pas de la même prééminence n'a rien d'objectif? C'est du reste ce dont témoigne le titre du $\$ 36$ des Ideen, qui commence par le «vécu intentionnel » pour aussitôt ajouter «le vécu en général », ce qui se laisse

12. Ibid., p. 113.

13. Ou dont il ne devait pas avoir connaissance, plus probablement.

14. F. Brentano, Psychologie du point de vue empirique, trad. de M. de Gandillac et éd. J.-F. Courtine, Paris, Vrin, 2008, p. 93. 
comprendre, par contraste, comme étant le vécu non-intentionnel. De ce point de vue, Husserl tient pour acquis le caractère intentionnel du vécu (qui en est définitoire, du point de vue eidétique, sans préjuger de la relation du vécu à un existant réel, l'attitude naturelle tombant dès les $\$ 31$ et 32 sous le coup de l'épochè), mais il faut remarquer que cette définition se trouve immédiatement nuancée lorsqu'il considère le vécu en général, lequel comprend deux types d'éléments non-intentionnels : les «data de sensation (Empfindungsdaten) ${ }^{15}$, ainsi que les «sentiments sensibles (sinnlichen Gefühlen) ${ }^{16}$. L'affectivité, au même titre que la matière hylétique de la sensation, entre ainsi à titre de composante dans la cogitatio mais est ce qui, comme telle, demeure non-intentionnelle tant qu'elle n'a pas été « animée ». Le confirme le $\$ 37$ qui suit, où Husserl distingue l'objet simplement intentionné (la Sache) de l'objet « intentionnel complet ${ }^{17}$, doté de valeur affective (l'Objekt).

"Quand je suis tourné vers une chose pour l'évaluer, il est sans doute impliqué que je saisisse la chose ; mais ce n'est pas la chose simple, mais la chose évaluée ou la valeur (...) qui est le corrélat intentionnel complet de l'acte d'évaluation ${ }^{18}$.

Or soutenir que l'intentionnalité s'appuie en dernière instance sur du non intentionnel, n'est-ce pas raisonner en termes d'inclusion psychique (à titre de composante) et, partant, réintroduire le subjectivisme ? Aussi, contre Husserl lui-même, Sartre, pour assurer la victoire du "dehors", dissocie l'affect de la grammaire du " ressenti », c'est-à-dire pense l'affect (ce que je 《 ressens ») sur le modèle de la perception (ce que je « vois »), autrement dit intentionnalise ce qui de prime abord paraît non intentionnel. Le régime intentionnaliste ne saurait souffrir d'exception. La partie finale du texte de 1939 opère par là un renversement d'une radicalité exemplaire : la seule manière pour évider jusqu'au bout la conscience revient alors à objectiver l'affect, à le mettre, lui aussi, au dehors. Haïr autrui revient encore à « s'éclater vers lui », les réactions jugées « subjectives » s'arrachent de «la saumure malodorante de l'Esprit $\gg{ }^{19}$.

Cette objectivation est telle que l'affect ne désigne plus la manière dont le sujet se rapport au monde mais, une certaine manière " commune" de réagir au quotidien, ce qui caractérise la chose elle-même : je hais non parce que j'ai un "problème» interne, mais parce que l'objet est lui-même haïssable. Ou comme l'écrit Sartre dans une formule frappante : "C'est une propriété de ce masque japonais que d'être terrible, une inépuisable, irréductible propriété qui constitue sa nature même, - et non la somme de

15. E. Husserl, Idées directrices pour une phénoménologie, trad. P. Ricœur, Paris, Gallimard, 1950, p. 117.

16. Ibid., p. 118.

17. Ibid., p. 120.

18. Idem.

19. J.-P. Sartre, La Transcendance de l'ego, op. cit., p. 112. 
nos réactions subjectives à un morceau de bois sculpté $»^{20}$. Ce passage peut cela dit s'interpréter en deux sens. Selon le premier, la qualité " haïssable » est cause de l'affect que je nomme « haine». Mais cette lecture, si elle tire son incontestable solidité du texte tel qu'il est formulé, à trop en respecter la lettre, risque fort d'en heurter l'esprit. Elle reconduirait en effet le dualisme entre une extériorité positive (douée de propriétés objectives) et une intériorité tout aussi positive (quoiqu'empruntant son contenu à l'extériorité). Cette position, comme nous allons le voir tout de suite, relève d'un empirisme que Sartre ne saurait accepter. Le second sens présente quant à lui l'avantage de suspendre le partage entre sujet et objet et d'annuler le problème qui consiste à se demander : mais quelle est la part réelle de ce que je perçois, et quelle est celle, subjective, qui à mon insu se glisse dans le perçu ? Autrui est-il haïssable car je le hais (projetant sur lui ce que je ressens) ou est-ce au contraire parce que je le hais qu'il est haïssable (« captant » une certaine propriété) ? De ce point de vue, si Sartre objective l'affect, c'est, semble-t-il, au sens où cette externalisation ne se dégage plus par rapport à quelque chose qui serait de l'ordre de l'intériorité : le masque terrible (à l'instar d'un soleil radieux, d'une journée agréable, d'un homme sympathique) figure le lieu d'indistinction entre le sujet et l'objet - il n'est pas légitime, encore une fois, d'interroger ce qui relève de l'un ou de l'autre, sauf à payer le prix exorbitant d'une réification du premier.

Ainsi, à plus d'un titre, la radicalisation de l'intentionnalité, tant en extension (l'affect y passe) qu'en intension (l'objet précède le percept), peut être présentée comme la réactivation d'une certaine tradition du «sens commun », celle de Thomas Reid, contre une certaine forme de réalisme indirect, où la donation des objets extérieurs se fait monnayant des idées intermédiaires : la déréalisation de la conscience (le fait qu'elle soit soustraite à l'ordre de la res) paraît constituer la seule solution possible pour défaire le problème de la représentation. Corrélativement, Sartre, ayant découplé le sujet de l'intériorité, désactive le problème qui consiste à savoir ce que nous avons en commun ; autrement dit, il déconstruit les conditions mêmes qui sont constitutives du problème du solipsisme.

\section{L'intentionnel, par-delà les sensations}

Mais il faut admettre que l'on voit mal quelle raison incite Sartre à rejeter de façon cavalière l'empiriocriticisme, le psychologisme et le néokantisme. Nous l'avions annoncé, l'imprécision du syntagme «philosophie digestive » doit sans doute être imputée à la valeur paradigmatique qui lui est conférée et à la stratégie sartrienne qui consiste à avancer ses pions en se donnant un ennemi si flou et démesuré qu'il en vient à désigner toute philosophie pour laquelle le rapport entre sujet et objet repose sur 
un dispositif de médiation. À cette difficulté s'ajoute la juxtaposition en apparence gratuite de traditions qui ont été historiquement en lutte, i.e. l'empiriocriticisme et le néo-kantisme. Or nous voudrions montrer dans ce qui suit que cela n'a rien d'anodin, dans la mesure où toutes deux partagent selon Sartre un même présupposé, i.e. la sensation ou le divers sensible, à savoir les Empfindungsdaten non intentionnelles dont faisait mention le $\$ 36$ des Ideen. Car si Sartre externalise la conscience, il évacue également l'idée que le dehors soit quelque chose qui doive être conquis. En d'autres termes, l'externalisme sartrien a pour ambition de rompre avec le problème de la constitution.

Gardons-nous pour le moment d'évaluer la justesse de la critique que Sartre adresse à l'empiriocriticisme car, de toute évidence, ce dernier en avait une connaissance de seconde main, et l'important ne se joue pas là. En effet, Sartre n'a aucune prétention exégétique, mais ce qui est significatif dans ce qu'il fustige sous le nom d'empiriocriticisme, c'est le fait que ce courant de la seconde moitié du XIX ${ }^{\text {ème }}$ siècle, qui se sert de Hume comme levier dans la critique qu'il mène de la Ding an sich kantienne, nourrit le projet de réduire le monde aux sensations. Sartre est à cet égard tributaire de l'interprétation qui en est déjà faite par les contemporains d'Ernst Mach et de Richard Avenarius, et qui consiste à y lire une forme de réactivation de l'idéalisme berkeleyen. Cette lecture subjectiviste du donné empiriocriticiste avait été notamment celle de Vladimir Ilitch Lénine en 1909, dans son Matérialisme et empiriocriticisme, lequel créditait Mach de concevoir le monde entier comme «ma » représentation ${ }^{21}$ et de proposer une théorie qui, en dépit des protestations réitérées du physicien, ressortissait au solipsisme. Mais c'est surtout par le biais de Husserl que le propos sartrien paraît s'éclairer. Husserl marquait en effet sa dette à l'égard de Mach, concevant le descriptivisme machien et l'exigence anti-métaphysique de retour au donné qui en est constitutive comme une forme de proto-phénoménologique que viendrait parachever la "science rigoureuse », ce dont atteste la boutade significative, du $\$ 20$ des Ideen («si par "positivisme" on entend l'effort, absolument libre de préjugé, pour fonder toutes les sciences sur ce qui est "positif", c'est-à-dire susceptible d'être saisi de façon originaire, c'est nous qui sommes les véritables positivistes »).

Or en dépit de cet appel commun à une critique de la métaphysique, Husserl adresse un double reproche à l'empiriocriticisme ${ }^{22}$. Le premier

21. Voir sur ce point Lénine, Matérialisme et empiriocriticisme: Note critique sur une philosophie réactionnaire (1909), préface et trad. d'A. Cervetto, Montreuil-sous-Bois, Éditions Science Marxiste, 2009, p. 40 : « Si, d'après Mach, les corps sont des "complexes de sensations" ou, comme disait Berkeley, des "combinaisons de sensations", il s'ensuit nécessairement que le monde entier n'est que représentation ».

22. Sur les rapports de Husserl et de Mach, voir H. Lübbe, «Positivismus und Phänomenologie: Husserl und Mach", in Bewustsein in Geschichten. Studien zur Phänomenologie der Subjectivität, Freiburg, 1972, p. 33-62 ; D. Fisette, «Phenomenology and Phenomenalism: Ernst Mach and the Genesis of Husserl's phenomenology », in Axiomathes 22 (1):53-74, 2012; et l'incontournable ouvrage de M. Manfred Sommer, Evidenz im Augenblick, Suhrkamp, 1987. 
se trouve consigné dans les cinquièmes Recherches logiques, notamment au $\$ 11^{23}$. Il vise l'amalgame entre le contenu de la sensation (la donnée hylétique) et l'objet de la sensation (le contenu intentionnel). En toute rigueur, ce que Husserl estime indéfendable dans cette théorie, c'est l'incapacité de cette dernière à restituer la transcendance de l'objet, à penser en termes d'inclusion réelle ce qui relève de l'ordre de la relation intentionnelle. Un tel reproche, dans le manque d'objectivité qu'il y diagnostique, reconduit en dernière instance celui qui lui a été inlassablement adressé, à savoir le subjectivisme dont est porteur, de manière plus générale, le paradigme empiriste. De ce point de vue, pour Sartre, l'empiriocriticisme vaut moins comme tradition constituée que comme le symbole de tout empirisme qui, au-delà du débat précritique qui en était définitoire au $\mathrm{XVII}^{\mathrm{e}}$ siècle et qui portait sur la question de savoir si l'intégralité de la connaissance pouvait être tirée de la seule expérience ou si la réflexion constituait une seconde source, est conduit, du fait même du point de départ qu'il se donne, i.e. la sensation, à manquer la transcendance du monde.

Mais à y regarder de plus près, tout se passe comme si Husserl avait tendu le bâton pour se faire battre. En effet, nous avions vu au $\$ 36$ des Ideen que l'affect se retrouvait, au même titre que la sensation, du côté des data hylétiques. La hylé est réelle mais non intentionnelle, et, on le sait, elle n'ouvre au champ de l'objectivité qu'une fois animée, rendue intentionnelle par un acte spécifique que Husserl nomme la noèse. Or qu'est-ce que cela signifie sinon que ce qui se joue ici, c'est le problème kantien de la constitution de l'objet, à savoir celui où le sujet se met en dehors du monde, le détruit depuis le lieu utopique (au sens étymologique de ce qui n'est nulle part) du transcendantal pour, une fois les yeux ouverts, s'étonner de le retrouver intact? À cet égard l'assomption de la hylé débouche sur une concession nouvellement faite à l'empirisme et, plus largement, à la "philosophie digestive ». D'une part, cela revient à vouloir faire sortir le transcendant de l'immanent (n'oublions pas la «philosophie douillette de l'immanence » en clôture du texte), à constituer le noème transcendant (irréel et présomptif, ainsi qu'en témoigne la réduction de l'objet au pôle inépuisable d'esquisses et de synthèses subjectives) à partir de l'immanence du vécu (réel et inclusif), i.e. à s'incliner à son tour devant l'illusion selon laquelle il est tout à fait possible, en se repliant sur la subjectivité, d'en faire sortir, comme l'écrit Sartre, « un objet transcendant en lui conférant la plénitude impressionnelle ${ }^{24}$. Autrement dit, de même que la hylé est ce

23. E. Husserl, Recherches logiques, Tome 2, Deuxième partie : Recherches III, IV, et V, trad. H. Elie, A. Kelkel, R. Schérer, Paris, PUF, 1961. Cette analyse, appliquée à Brentano, vaut pour Mach. Cf. E. Husserl, Logique formelle et logique transcendantale. Essai d'une critique de la raison logique (1929), trad. S. Bachelard, Paris, PUF, 1957, p. 226, où Husserl s'en prend $(\$ 62)$ au "positivisme» de Mach qui réduit les objets à des complexes, "réglés empiriquement, de data psychiques (des "sensations") ».

24. J.-P. Sartre, L'Être et le Néant. Essai d'ontologie phénoménologique, Paris, Gallimard, 1943, p. 27. 
par quoi il y a intentionnalité sans être elle-même intentionnelle, de même constitue-t-elle une forme de "proto-transcendance » qui ne ressortit pas elle-même de la transcendance. Comme l'écrit Vincent de Coorebyter, «c'est ici la solution qui fait problème : on arrime l'objet au sujet pour mieux arrimer le sujet à l'objet, on encapsule le monde dans la sensation avant de chercher à dilater la sensation pour retrouver le monde. À gager l'extériorité sur l'intériorité, à reporter sur la hylé la charge du non-moi en général, la phénoménologie risque de verser dans le phénoménisme $»^{25}$. Du reste sera-ce dans L'Être et le Néant la conclusion qu'en tirera Sartre, repérant chez Husserl une identification entre l'esse et le percipi, qui se verra qualifiée de « monisme du phénomène $»^{26}$.

D'autre part, la hylé introduit dans la conscience un bout de réel que celle-ci ne s'est pas donnée, ce en quoi la hylé mesure la faiblesse de l'intentionnalité. Elle forme à ce titre un reliquat de l'empirisme classique, lequel fait de l'esprit l'effet dont le réel est la cause. Mais ce concept relève également de l'idéalisme, car de même que le Noûs chez Anaxagore, après avoir organisé la matière informe, délivre le cosmos, de même la hylé n'a de valeur qu'au regard de sa reprise ultérieure : c'est une fois configurée qu'elle peut livrer quelque chose comme un l'objet.

La sensation est donc ce que partagent l'idéalisme et l'empirisme. En effet, l'idéalisme s'autorise de cette sensation catégorialement nue pour mieux justifier l'intervention d'un agent méta-empirique de synthèse. En cela, Husserl demeure tributaire du concept transcendantal de Gegebenheit, où le donné comme simplement donné (au sens de ce qui advient, par la sensibilité) s'avère " posé », « suscité » par la réflexion, tirant tout son sens de ce sur quoi il ouvre, à savoir l'objet, qui ne peut être que tel ou tel, c'està-dire dont la détermination repose sur une synthèse catégoriale. Aussi doiton en déduire que si la sensation (pure) n'a de sens que relativement à la catégorialité (qui en fait une perception - laquelle est de ce point de vue toujours perception d'objet), c'est alors que l'intentionnalité doit être dissociée du schème hylémorphique et ne plus constituer le revers d'un envers hylétique. Partant, Sartre déboute l'intentionnalité de la hylé qui en légitimait le concept. Seulement ainsi pouvons-nous prendre la mesure du réalisme de la lecture de l'intentionnalité. Dire que Sartre détourne les Ideen, que par son réalisme il fournit une lecture hétérodoxe, voire fautive, du concept d'intentionnalité, c'est encore trop peu dire. Il est nécessaire d'aller plus loin: Sartre le subvertit de bout en bout. En refusant de fonder l'intentionnalité sur du non intentionnel, Sartre rompt avec le problème de la constitution de la transcendance. Cessant d'appeler à sa constitution, le réel,

25. V. de Coorebyter, Sartre face à la phénoménologie, op. cit., p. 55. Du reste J. Patočka décèle le même problème et distingue la réduction de l'épochè (Qu'est-ce que la phénoménologie?, édité et traduit par Erika Abrams, Grenoble, Million, 2002, p. 208 : "Comment le vécu, originairement donné à soi-même dans la réflexion, s'y prend-il pour faire apparaître une transcendance du côté objectif ? C'est incompréhensible »).

26. J.-P. Sartre, L'Être et le Néant, op. cit., p. 11. 
dans sa transcendance, devient ce qu'on se contente d'attester. En d'autres termes, la hylé est la véritable pierre d'achoppement de l'intentionnalité, et non sa condition de possibilité. Se débarrassant de la philosophie de la représentation ${ }^{27}$, Sartre se défait de la conception intellectualiste de la perception comme connaissance d'objet et, partant, de la question de savoir ce qui permet de passer du divers sensible à l'ordre de l'objet. Par conséquent, s'il ne peut être question de synthèse chez Sartre (et, partant, d'agent de synthèse), c'est parce que le non-synthétisé qu'est le matériau impressionnel fait lui-même figure de mythe : répudier le synthétisable (non au sens où il y aurait quelque chose qui puisse ne pas l'être, mais au sens où il faut une forme de "sous-objet», qui ne ressortisse pas encore au régime véritable de l'objet), c'est répudier l'idée totale de synthèse.

\section{Le psychologisme et la naturalité du sujet}

Venons-en au second reproche que Husserl adresse à l'empiriocriticisme : le psychologisme. Cette « catégorie », devenue centrale au tournant du $\mathrm{XX}^{\mathrm{e}}$ siècle, désigne la réduction des objectités sémantiques, censées être invariables, aux actes psychiques, et la subordination du logique (ce qui doit être pensé) au psychologique (la manière dont, naturellement, on pense). Le psychologisme consiste en effet à naturaliser la pensée mais, par-là même, à raturer l'exigence d'objectivité et d'universalité à laquelle la logique prétend. Sous cet angle, tout se passe comme si Husserl rejouait avec Mach l'opposition de principe entre Hume et Kant. Alors que pour le premier l'enchaînement des idées se fait par association, contiguïté et ressemblance, autrement dit que le mode de réglage des idées entre elles est éminemment factuel et relatif ${ }^{28}$, et en cela indissociable du scepticisme, pour le second il s'agit de sauver l'objectivité de la science par le recours à la légalité a priori du catégorial. En un passage célèbre de sa Logique, que Husserl reprend à son compte au $\S 19$ de ses Prolégomènes à la logique pure, Kant exhibe les limites qui sont celles-là mêmes de cette psychologie empirique dont le seul mérite est, tout au plus, de faire ressortir la manière dont l'entendement fonctionne et les lois subjectives qui règlent l'enchaînement des idées, sans jamais, en en restant là, atteindre à la nécessité de la loi. En cela, Husserl se range aux côtés de Kant réfutant toute une approche empiriste de la logique qui, prétextant du fait que les

27. Cf. G. Deleuze, «Il a été mon maître », L'île déserte. Textes et entretiens 1953-1974, édition préparée par D. Lapoujade, Paris, Les Éditions de Minuit, 2002, p. 110-111 : «Toute sa philosophie s'insérait dans un mouvement spéculatif qui contestait la notion de représentation, l'ordre même de la représentation : la philosophie changeait de lieu, quittait la sphère du jugement pour s'installer dans le monde plus coloré du "préjudicatif", du "subreprésentatif" ".

28. Pour aller plus loin, voir J. Benoist, « Le naturalisme : avec ou sans le scepticisme ? Après Hume », Revue de métaphysique et de morale, 2003/2 (nº 38), p. 127-144. 
lois logiques dépendent de l'acte psychique qui en est porteur, conclut de la dépendance effective de la légalité logique à l'égard de l'activité psychologique à la dépendance idéelle (ou sémantique) de la première à la seconde. En effet cette fondation subjective de la vérité logique ne peut que succomber à une forme de « contradiction performative » dans la mesure où l'empirisme "supprime la possibilité d'une justification rationnelle de la connaissance médiate et supprime par-là même sa propre possibilité, en tant que celle d'une théorie scientifiquement fondée ${ }^{29}$. Bref, l'empirisme se contredit, tout se passant comme s'il accordait un sens objectif au logique afin de s'établir comme théorie, pour immédiatement après biffer l'objectivité de ce sens.

De fait, Husserl décèle cette même confusion entre le réel et l'idéel dans l'empiriocriticisme de Mach et d'Avenarius. Le chapitre IX est en effet entièrement consacré au «principe d'économie ». Certes, celui-ci a ceci de novateur qu'il conçoit la connaissance comme une fonction vitale et refuse la dichotomie entre praxis et scientia: le concept, comme le soutient Mach dans Erkenntnis und Irrtum, constitue un abrégé de l'expérience, dont il met en exergue un aspect. Cela rend possible une économie en "énergie ${ }^{30}$. Mais cette refondation empirique de la logique, parce qu'elle est devenue populaire dans un contexte marqué par l'écrit que Darwin publie en 1859, De l'origine des espèces, requiert à son tour un examen critique. Certes la Denkökonomie joue un rôle indéniable dans l'établissement de l'anthropologie psychique et épistémologique, ouvrant une «sphère d'investigations étendues, fructueuses et instructives » précisément parce que le «domaine du psychique» constitue une partie «du domaine de la biologie $»^{31}$, mais il n'en reste pas moins qu'elle est incapable de fonder la logique pure et la théorie de la connaissance. En effet, il s'agit non pas d'un principe explicatif mais d'un résumé de faits où s'atteste l'adaptation du vivant à son environnement (il porte mal son nom). En outre, la psychologie, si elle se voit réduite à ce " principe » téléologique, se verra incapable d'aller plus loin dans l'explication des fonctions psychiques.

Quelle conclusion en tirer? Que la fondation biologique de la logique présente le même tort aux yeux de Husserl que la fondation sensualiste et que le reproche qui est adressé à Mach n'a au fond rien de bien spécifique : ce dernier, à l'instar de ses prédécesseurs et surtout de Hume, naturalise la pensée logique, rabattant l'idéel sur le factuel. Bien sûr, on pourrait rétorquer que le terme de «psychologisme » que Sartre emploie au début de son texte sur l'intentionnalité n'a pas de sens technique : replacé dans l'économie du texte, il désignerait tout ce qui relève de la «psychologie », laquelle pense la

29. E. Husserl, Prolégomènes à la logique pure, trad. H. Elie, A. Kelkel et R. Schérer, Paris, PUF, 1959, p. 94 (appendice).

30. Schlick formulera une critique similaire dans sa Théorie générale de la connaissance (1925 pour la seconde édition), trad. et présenté par C. Bonnet, Gallimard, NRF, Paris, 2009, p. 159.

31. Ibid., p. 217. 
conscience sur le mode de la chose. Aussi juste soit-elle, cette objection présente néanmoins l'inconvénient de ne pouvoir justifier la juxtaposition sur laquelle s'ouvre le texte de 1939. Or il faut souligner que lorsque Sartre prend connaissance à Berlin des Recherches logiques, c'est dans le cadre d'une attaque en règle de toute fondation empirique de la logique: l'empiriocriticisme, en dehors de la philosophie des sciences (Duhem a par exemple salué la Mécanique et Mach a laissé sa marque, introduisant la méthode historico-critique dans la rationalité scientifique), rend un son, il faut bien l'admettre, barbare.

\section{Le « jamais-né » : la renaissance du sujet métaphysique}

Aussi suggérons-nous que ce qui peut gêner Sartre dans le «psychologisme» est moins le fait que s'atteste là encore un ratage de la transcendance, fût-elle logique, que ce qu'il implique de façon sous-jacente. En effet, quel a été le fil directeur opposé qu'a suivi Husserl dans l'établissement programmatique de la logique pure si ce n'est celui de la naturalisation de l'esprit, que Hume a véritablement initiée ? Naturaliser l'esprit, c'est dire que l'esprit fait partie de la nature, qu'il est $d u$ monde mais non $a u$ monde. Mais s'il est naturel, alors rien en lui ne le différencie fondamentalement, par une propriété qui en serait constitutive, et il faut alors en conclure que l'activité scientifique (et la pensée en général) est en continuité avec l'activité animale (ce qu'on appellerait « l'instinct»), ce que du reste Husserl épinglait dans ses Prolégomènes. Or c'est précisément ce que Sartre ne peut admettre. Si la conscience n'est rien à proprement parler, elle ne se confond pas pour autant avec la phusis. C'est même tout le contraire. Sartre avance un dispositif discontinuiste (l'animal est le grand absent de sa philosophie), dont le texte de 1939 constituait la première ébauche et que L'Être et Néant amène en 1943 à sa complétion.

Sartre, cessant, dans la Transcendance de l'Ego, de chercher à établir la légitimité de sa pensée en recourant à la phénoménologie historiquement établie, élimine le reste de res cogitans dont l'ego transcendantal était le dépositaire: Husserl avait en effet réalisé en site phénoménologique l'aperception kantienne et offrait quelque chose comme un «moi pur», un Ichprinzip qui, quoique portant sur tout vécu, ne s'identifiait pourtant à aucun d'eux. Mais parler comme le fait Husserl au $\$ 57$ des Ideen de «transcendance au sein de l'immanence» revenait pour Sartre à injecter dans la conscience «translucide» de l'opacité, à penser l'ego à la fois comme principe et comme dérivé, comme constituant et comme constitué. En cela, l'ego husserlien était un hybride qui résultait d'une illusion rétrospective, la réflexion projetant en amont d'elle une instance égologique qui lui servît à la fois d'objet (rapport à soi) et de condition de possibilité (soi qui se rapporte à). En effet, pour Sartre, le cogito à dire « je » se rate et se pense comme une chose. La réflexion conduit la conscience à se saisir sur le mode de l'objet. Soit on est dans la vérité de la conscience et le sujet ne 
se saisit pas car il n'est pas dans l'auto-thématisation, soit le sujet se saisit mais se rate dans cette saisie comme sujet: Tirésias l'avait prédit, Narcisse, symbole de la prétention (vouée à l'échec) à se connaître soi-même, n'atteindrait un grand âge qu'à condition de renoncer à se connaître.

Mais ce qui révèle de façon éclatante ce statut encore métaphysique que Sartre tient à conserver à la conscience, c'est l'opération qui consiste à éliminer l'ego transcendantal sans pour autant renoncer au transcendantal. L'ego est évacué de la conscience, il n'en est plus, comme l'écrit Sartre, un « habitant $»^{32}$, de sorte que ce qui se délivre là est un champ transcendantal anonyme, qui ne puisse plus dire « je » sans se prendre pour ce qu'il n'est pas. Le concept de réflexion, comme on l'a vu, est le levier qui permet de purifier le transcendantal de tout « Je» : la conscience est non positionnelle d'elle-même, dans un rapport irréfléchi et immédiat à elle-même, ce que du reste réaffirmera l'Être et le Néant sous la dénomination de «cogito préréflexif $\iota^{33}$, tandis que l'ego résulte d'une constitution via la réflexion, i.e. constitue un objet présomptif (au même titre que le noème husserlien), unité idéale des états et des actions. Sartre fait tomber le Je sous le coup de la réduction phénoménologique, mais conserve le transcendantal: préfigurant la thèse de 1943, la Transcendance de l'ego fustige déjà cette «tendance dangereuse de la philosophie ${ }^{34}$, qui est notamment celle de l'empiriocriticisme, qui consiste à vouloir déterminer le transcendantal, à se demander ce qu'il est. Qui, en d'autres termes, passe du droit au fait, et voulant «réaliser les conditions de possibilité déterminées par la critique $»^{35}$, pense le transcendantal sur le mode de la genèse de la conscience empirique. Or le transcendantal n'est rien dont on puisse éclairer la genèse dans la mesure où il n'a rien d'empirique : ce refus de l'empirisme et de la naturalité de la conscience qui lui est corrélée se cristallise donc dans la mise en place d'un transcendantal impersonnel dont à proprement parler on ne peut rien dire sans risquer de le perdre en le confondant avec l'objet égologique. À cet égard, on remarque que Sartre reprend le transcendantal kantien, mais qu'il le dépouille du «Je Pense» qui, posait l'Analytique transcendantale $(\S 16-$ - De l'unité originairement synthétique de l'aperception »), «doit pouvoir accompagner toutes nos représentations »: sans ego, le transcendantal n'en reste pas moins ce qui est nécessaire (il « doit» être pour qu'il y ait expérience) et dépourvu d'empiricité (il doit « pouvoir »). Mais en même temps, Sartre veut passer du droit au fait, ne plus faire du transcendantal un simple «idéal » mais un «fait absolu » ${ }^{36}$, " accessible à chacun de nous » une fois réalisée l'opération de réduction.

32. J.-P. Sartre, La Transcendance de l'ego, op. cit., p. 13.

33. Ibid., p. 16.

34. Ibid., p. 14.

35. Id.

36. Ibid., p. 18. Sur ce point, voir J.-M. Mouillie, Sartre. Conscience, ego et psychè, Paris, PUF, 2000, et notamment le sous-chapitre qui porte sur «l'antinomie de l'ego », p. 53-55. Mais aussi P. Cabestan, Qui suis-je ? Sartre et la question du sujet, Paris, Hermann, 2015, p. 19-45, sur le statut du cogito. 
De toute évidence Sartre se trouve embarrassé par la réduction, pris en tenaille par deux impossibilités. D'un côté, il ne peut se résoudre à mettre le monde de l'attitude "naturelle " entre parenthèses, dans la mesure où cela reviendrait d'une part à relativiser le monde par rapport à une conscience érigée en absolu, et d'autre part à en effectuer l'opération depuis le point de vue de ce qui aurait dû être lui-même réduit, i.e. l'ego transcendantal. Mais d'un autre côté, tout se passe comme si Sartre ne pouvait accepter cette naturalité de la conscience, en faire un être intramondain. Autrement dit, se fait jour chez Sartre une tension entre l'exigence d'accepter l'attitude naturelle (pour sauver le monde), et tout en même temps l'impossibilité d'y agréer pleinement (pour sauver la conscience). La conscience ne peut être quelque part : ce serait en effet la concevoir sur le mode de la chose, qui se contente d'être ce qu'elle est, quand bien même elle serait hors-monde et dite "transcendantale ». Or il s'agit pour Sartre de casser ce modèle " chosiste » et réifiant du sujet. Sartre exige que l'on tienne les deux bouts : que la conscience ne soit pas quelque part, localisable, simple partie de la nature, mais qu'elle ne soit pas non plus quelque chose qui soit hors-monde. La conscience ne peut être indépendante de l'être du monde (elle est rapport, sans intériorité), mais elle ne peut non plus en dépendre (elle n'est pas une chose, avec son extériorité positive). Elle ne saurait être dans le monde, ni lui être extérieure, de sorte qu'une seule conclusion puisse être tirée: la conscience ne sera rien. Comme on le sait, dans l'Être et le Néant le sujet se " définit» par son inadéquation principielle à lui-même, étant pure négation, au contraire de l'en-soi compact des choses, bloc de positivité. Et ce « rien », semble-t-il, libère conceptuellement l'aporie : le sujet n'est plus quoi que ce soit que l'on puisse indexer sur un « ici» ou un «ailleurs ». Mais partant, Sartre offre un concept de dehors pour le moins étrange, qui ne soit plus le dehors d'aucun dedans (l'intentionnalité permettait d'étayer cette thèse), ni non plus le dehors du monde (le refus du "psychologisme» et du naturalisme qui en est l'arrière-plan philosophique). Mais soutenir que la conscience n'a rien de naturel et qu'à la fois elle n'est que rapport à la nature, sur quoi cela débouche-t-il ?

Assurément, on peut reprocher à Sartre d'en rester à l'attitude naturelle et de "succomber» au réalisme ${ }^{37}$. Sartre établit en effet une dissymétrie principielle entre l'en-soi et le pour-soi. Si, à rebours de la Ding an sich kantienne, qui est exclusive de l'Erscheinung, l'en-soi sartrien (ou «être transphénoménal ») n'est pas trahi par son paraître, il n'en jouit pas moins d'une antériorité logique sur lui. La négation qu'est le pour-soi, on le sait, se prélève sur la positivité de l'en-soi : on ne peut nier que ce qui est niable, la négation ne saurait précéder ce dont elle est la négation. S'opposant à la contemporanéité logique de la dialectique hégélienne entre être et non-être,

37. R. Barbaras, "Désir et manque dans L'Être et le Néant: le désir manqué », in Sartre. Désir et liberté, coordonné par R. Barbaras, Paris, PUF, 2005, p. 136 : «En parlant d'en soi, Sartre introduit donc d'emblée, sur un mode finalement réaliste, une scission, entre l'être et le phénomène (...). En ce sens, il s'agit d'une phénoménologie sans phénomènes ». 
Sartre soutient que le " non-être n'est pas le contraire de l'être, il est son contradictoire», ce qui «implique une postériorité logique du néant sur l'être puisqu'il est l'être posé d'abord puis nié $)^{38}$. L'ens ne surgit plus $e x$ nihilo, mais c'est le nihil qui surgit ex enti.

Or cette priorité du positif reste stérile et impuissante à se poser, car c'est depuis la négation, qui introduit un écart, que la positivité peut être affirmée. Si la positivité de l'en-soi jouit d'une antériorité logique, elle ne tire tout son sens que de ce «ver » qu'est le pour-soi, i.e. la positivité accède à sa vérité depuis le point de vue de sa négation. Cette situation intermédiaire, un peu bancale, n'était-elle pas déjà lisible dans le sous-titre même de l'Être et le Néant, "essai d'ontologie phénoménologique ", qui, exprimant le souci d'éluder tout risque de "phénoménisme » (au sens large de ce qui rabat l'esse sur percipi), mais aussi toute une tradition qui place l'être à part de la perception, établit la priorité onto-logique de l'en-soi sur le pour-soi mais, inversement, la priorité phénoméno-logique du pour-soi sur l'en-soi ? En effet, si la conscience n'est pas productrice d'être dans la mesure où elle se prélève comme néant sur l'ens compact des choses, elle n'en joue pas moins un rôle primordial en ce qu'elle rend possible le surgissement de ce qui ontologiquement la précède. L'en-soi, incapable de venir à la présence par lui-même, dépourvu de toute capacité d'automanifestation, de Selbstgegebenheit, a besoin d'autre chose à titre de condition de possibilité de phénoménalisation. Sartre, envers et contre tout, demeure profondément husserlien, eu égard à la fonction «phénoménalisante » qui est dévolue au pour-soi : si, comme nous l'avons analysé, il purifie l'intentionnalité de la problématique de la constitution, il n'en conserve pas moins l'idée que l'en-soi requiert pour se manifester une structure d'apparaître. En d'autres termes, Sartre substitue à la constitution noématique la constitution, ainsi que la qualifie Jean-Marc Mouillie, «dévoilante ${ }^{39}$.

Cette manière de prêter au pour-soi seul le pouvoir de révéler l'en-soi ne participe-t-elle pas de la démarche régressive qui consiste à passer du donné à ce qui donne, de l'expérience à sa condition de possibilité, de ce qui paraît à celui grâce à qui il y a à apparaître ? Cela signifie à tout le moins qu'il n'y a de donné que pour un sujet et que le sensible constitue le strict corrélat d'une structure de phénoménalisation qui n'est autre que la conscience. Qu'il n'y ait pas de sensible qui puisse se précéder avant le surgissement du sujet, pas de donné qui ne soit donné à quelqu'un. De ce point de vue, le phénoménologue arrive toujours déjà trop tard: l'en-soi, comme en-soi, est toujours et irrémédiablement perdu. Ainsi, le réalisme sartrien, que ce soit pour le blâmer ou le louer, ressemble à la surface émergée d'un iceberg. Le revers du réalisme de Sartre, c'est son kantisme. Il ne s'agit pas d'un réalisme transcendantal (car le cogito n'est pas une

38. J.-P. Sartre, L'Être et le Néant, op. cit., p. 49.

39. J.-M. Mouillie, «S. et H. : une alternative phénoménologique? », in Sartre et la phénoménologie, textes réunis par J.-M. Mouillie, ENS-LSH Éditions, 2000, p. 94. 
res) ${ }^{40}$, ni d'un idéalisme transcendantal (car il ne s'agit pas de constituer le transcendant à partir du vécu), mais, si l'on veut bien nous passer l'expression, d'un nihilisme transcendantal (au sens où la condition de possibilité de l'expérience équivaut au néant du pour-soi, à un nihil). Là où Husserl constitue l'objet irréel à partir de la hylé réelle, Sartre constitue le phénomène réel à partir du sujet irréel. L'un dégage du transcendant comme noème à partir de la plénitude impressionnelle de la conscience absolue, l'autre dégage du phénomène à partir de la vacuité de la conscience. Ce transcendantalisme se révèle donc singulier, puisque dissocié de ce pour quoi il avait été établi, à savoir, pour contourner le scepticisme de Hume, la fondation de l'objectivité via la légalité du catégorial : en effet, tout se passe comme si Sartre mettait ce dispositif transcendantal au service non de la science, mais de l'éthique (masochisme, amour, sadisme : autant de façons de jouer avec soi-même le jeu de ce que je ne suis pas, sur le mode de l'objet, d'occulter mon inconditionnelle libertét ${ }^{41}$, ce que Sartre nomme «mauvaise foi »), dans une sorte d'appel à la responsabilité de l'ordre du catéchisme laïcisé.

\section{Conclusion : le mythe de l'extériorité}

Dans cette phénoménologie «réaliste », l'enjeu était obvie: que la pensée puisse quitter le giron de ses représentations et, pour la première fois dans son histoire, avoir le courage de mordre sur le réel. Ce qu'exprime Sartre dans un article de 1961 consacré à Merleau-Ponty : " J'eus l'obscure souvenance d'une pensée que je n'ai peut-être jamais eue : la vérité traine par les rues, dans les fabriques et, mise à part la Grèce antique, les philosophes sont des eunuques qui ne lui ouvrent jamais leur porte $»^{42}$. Mais cette morsure était en réalité pleine d'ambiguïtés, l'immédiateté creusée de médiations. La conscience était certes mise en rapport direct avec les choses, sans intermédiaires, qu'il s'agisse de sensations ou de représentations. Ces choses, si elles n'étaient point déduites de la subjectivité, présentaient néanmoins un visage humain, tributaires qu'elles étaient de la fonction dévoilante du pour-soi. Voulant mettre la vérité dehors, Sartre la faisait à son insu rentrer par la porte de derrière. «L'Esprit-Araignée attirait les choses dans sa toile ${ }^{43}$, mais cette toile n'était-elle pas par la suite expulsée au dehors?

40. Husserl s'attaque à ce qu'il appelle le «réalisme transcendantal» de Descartes, qui consiste à amalgamer le moi transcendantal et le moi empirique de l'attitude naturelle, ce qu'atteste le passage dans la seconde des Méditations métaphysiques du cogito à la res cogitans (cf. Méditations cartésiennes, trad. E. Levinas et G. Peiffer, Paris, Vrin, 1947, §10, p. 52).

41. Cf. C. Poulette, Sartre ou les aventures du sujet. Essai sur les paradoxes de l'identité dans l'œuvre philosophique du premier Sartre, Paris, l'Harmattan, 2001.

42. J.-P. Sartre, Situations, IV. Portraits, Paris, Gallimard, 1964.

43. J.-P. Sartre, La Transcendance de l'ego, op. cit., p. 109. 
Le kantisme que nous y avons décelé procède par conséquent d'une inversion structurelle permanente et érigée en règle : mettre la conscience dehors c'est tout aussi bien transplanter ce qu'elle avait en elle. Le dehors est un terme en effet relationnel, qui tire son sens de ce à quoi il s'oppose : qu'est-ce qui pourrait être qualifié de dehors si ce n'est ce qui n'est pas dedans? Or, en absolutisant le dehors, en proposant un dehors qui ne soit l'envers d'aucun dedans, Sartre, avons-nous vu, n'a pas tant supprimé "l'intériorité » qu'il ne l'a déplacée. Tel est en effet le risque qu'encourt tout dualisme, toute relation qui se reporte tout entière sur l'un de ses termes. Dans ce report, elle entraîne avec elle l'autre terme. Ce kantisme structurel chez Sartre, surmonté d'un réalisme "métaphysique », se trouve retranscrit dans le concept même d'intentionnalité : désirant sortir $\mathrm{du}$ «corrélationnisme» kantien, Sartre, ayant cru s'en extirper par la déréalisation de la conscience, le reconduit sous la forme d'un remède qui en est en réalité le poison, i.e. l'intentionnalité, qui se fait pourtant toujours entre deux termes. De Brentano à Sartre en passant par Husserl, tout se passe comme si l'intentionnalité était progressivement délestée de son idéalité, que l'histoire du concept était celle d'une conquête de l'extériorité. À cet égard, l'externalisme radicalisé de Sartre a tout l'air de constituer l'envers d'un internalisme lui-même radicalisé, la «pensée du dehors » une "pensée du dedans " qui s'est retournée comme un gant, mais qui demeure au fond tributaire de cette division conceptuelle entre l'intérieur et l'extérieur qu'elle prétend dépasser. Ce renversement du dedans en dehors, où le dehors emporte avec lui ce dedans qu'il pose pour ensuite le raturer, paraît inévitable dès lors que la radicalité du réalisme sartrien se gage sur celle d'une position adverse rendue disproportionnée. Tel était le prix à payer à se donner pour ennemi une "philosophie digestive», dehors à la mesure de la caricature du dedans.

Il était pourtant une autre manière de procéder : non en conservant l'intentionnalité en se persuadant qu'il est possible de replier toute la relation sur l'un de ses relata, croyant outrepasser la grammaire même, dualiste, de l'intentionnalité, mais en mettant fin à la scission entre le sujet et l'objet. Autrement dit, en déconstruisant le partage, jugé métaphysique, entre le dedans et le dehors. Une telle piste était offerte, d'une part avec le concept d'image dans le premier chapitre de Matière et Mémoire d'Henri Bergson, que Sartre stigmatise dans son article de 1939, et que Bergson avait pourtant définie comme ce qui est à mi-chemin entre la chose du réaliste et la représentation de l'idéaliste ${ }^{44}$, comme ce qui ne peut être compris à partir de ce qu'elle a précisément pour tâche de neutraliser, à savoir la dichotomie entre sujet et objet, entre la pensée et le réel. D'autre part, l'empiriocriticisme d'Avenarius et de Mach, qui propose un concept de sensation qui ne fasse plus le jeu du dualisme et qui parvient à dépasser le

44. Pour une lecture minutieuse du premier chapitre, voir $\mathrm{C}$. Riquier, « $\mathrm{Y}$ a-t-il une réduction phénoménologique dans Matière et mémoire?", in F. Worms, Annales bergsoniennes II, Presses Universitaires de France, coll. » Épiméthée », 2004, p. 261-285. 
kantisme, i.e. à se débouter du concept transcendantal de Gegebenheit. Plutôt que de faire le jeu du dedans et du dehors, Mach affirmait, dans L'Analyse des sensations, qu'il n'y a pas « de dedans, ni de dehors -, pas de sensation à laquelle correspondrait à l'extérieur un objet différent d'elle ${ }^{45}$. Ce renvoi dos-à-dos du réalisme (avec l'objet indépendant) et de l'idéalisme (avec le primat du sujet) ne pouvait s'effectuer qu'une fois avoir rompu avec la trame kantienne. Si Mach refuse de mettre un pied dans ce qu'il appelle, dans Erkenntnis und Irrtum, « le pays du transcendantal », c'est bien parce qu'il s'agit de faire droit à l'expérience, et rien qu'à elle, sans y introduire ce dont elle est dépourvue, i.e. l'a priori, qui ne provient pas de la sensibilité, mais de cette seconde source qu'est l'entendement. En outre, l'empiriocriticisme refuse de valider la "révolution copernicienne» qui consiste à passer de la question de l'objet à celle de la faculté de connaître, à interroger non l'objet mais le rapport du sujet à l'objet. Cette démarche régressive vers les conditions de possibilité de l'expérience est pour Mach inacceptable, si tant est que ces conditions se situent en dehors du cadre de l'expérience. Bref, l'empirisme de Mach est un empirisme qui intègre le kantisme comme une donnée avec laquelle il faut composer (de même que Hume réveille Kant de son sommeil dogmatique, Kant, à son tour, réveille Mach de son réalisme naif), mais il est surtout cet empirisme qui a su dépasser le kantisme plutôt que de s'installer en lui comme dans un cadre paradigmatique.

C'était du reste ce qu'aurait pu entreprendre Sartre, qui avait été pourtant marqué par la lecture de Vers le concret, paru en 1932. Dans ce dernier, Jean Wahl écrivait que c'est «l'honneur de l'École empiriocriticiste souvent traitée d'une façon injuste par ceux qui se réclament du matérialisme dialectique, et c'est l'honneur de philosophes aussi différents qu'un James et qu'un Bergson d'une part, qu'un Russell de l'autre, d'avoir décelé les fausses positions de certains problèmes et dénoncé les pseudoproblèmes $\gg{ }^{46}$. Mais la phénoménologie exerçait alors sur son esprit une influence plus forte encore: Sartre, semble-t-il, croit sur parole Husserl lorsque celui-ci prétend radicaliser l'empiriocriticisme sous forme de phénoménologie. Or si l'on doit parler de radicalité, c'est au sens où, comme on l'a vu, Husserl remplit à la perfection le cahier des charges kantien, ce en quoi on peut bien s'interroger sur les droits que s'arroge une discipline qui, du moins à ses origines, est moins un descriptivisme pur qu'une manière de soumettre le donné au diktat du logos (la phénoméno-logie), quitte à déclarer par après qu'elle ne veut rien d'autre qu'effectuer un «Zurück zu den Sachen selbst ». Mais, du point de vue de la neutralité du donné, on peut douter des proclamations réitérées de Husserl qui loue Mach d'avoir inauguré une méthode sans l'avoir menée à son point d'accomplissement.

45. E. Mach, L'Analyse des sensations (1886), traduit de l'allemand par F. Eggers et J.-M. Monnoyer, préface de J.-M. Monnoyer, Nîmes, J. Chambon, 1996, p. 272.

46. J. Wahl, Vers le concret. Études d'histoire de la philosophie contemporaine, avant-propos par M. Girel, Paris, Vrin, 2004, p. 38-39. 
En somme, nous souhaitions prendre au mot Sartre lorsqu'il fustige la «philosophie digestive», et il faut en convenir que les choses se sont révélées être plus complexes qu'elles n'y paraissent. Là où Kant admet l'empirisme et l'idéalisme dans son criticisme, empruntant au premier la sensation brute et au second la synthèse catégoriale, Sartre rejette l'empirisme mais adopte le dispositif kantien de la chose en soi et du transcendantal, rejetant comme ennemi ce qui seul avait véritablement rompu avec le kantisme sous la forme de ce que William James avait appelé, dans son écrit posthume de 1912, un « empirisme radical». De toute évidence le horla du dedans poursuit son dehors comme son ombre. La «digestion», que Sartre attaquait dans son article de 1939, se retrouve en 1943 sous la forme d'un "schéma » qui structure le rapport entre la conscience et le monde. Là où la créature, dans sa déficience ontologique, aspire à retrouver la plénitude d'être du créateur, de façon similaire, mais par une inversion du dedans divin en dehors ontologique, le pour-soi est travaillé par une faim ontologique, condamnée à rester insatisfaite : faute d'être quoi que ce soit, parce qu'il est carence, béance, le pour-soi figure ce trou malheureux et affamé qui voudrait rejoindre, sans espoir possible, l'ensoi, réaliser la synthèse impossible du pour-soi avec un en-soi indigeste, autrement dit être sur le mode de ce qu'il n'est pas. En outre le sujet n'existe plus à part du monde, mais il refuse tout en même temps d'y entrer, ce qu'illustre la dichotomie entre le champ transcendantal anonyme (un sujet d'autant plus fort qu'il ne dit plus «Je ») et l'ego intramondain, qui se voit reportée dans L'Être et le Néant sur celle entre le «néant» de la conscience et le « moi » de la mauvaise foi.

Cette «pensée du dehors », qui met ainsi au dehors un sujet transfiguré qui ne peut plus se penser sur le mode cartésien du « je pense », qui est horsmonde tout en étant corrélé à ce monde, bref une pensée de la conscience déboutée de l'égologie cartésiano-husserlienne, a ainsi tout l'air, dans ce maintien d'un sujet méta-empirique (et qui ne joue plus le rôle d'agent de synthèse), d'un internalisme non surmonté. On peut bien s'interroger en retour sur une époque où nous croyons nous être débarrassés de la métaphysique et sur le sens d'une condamnation quasi-unanime et consensuelle, qui ne fait plus question alors qu'elle était motivée, chez Kant, par un état de crise de la philosophie. Le pathos qui entoure le dit "mythe » de l'intériorité s'est renversé en une dramaturgie de l'extériorité à outrance, sans qu'on se demande si, au fond, l'externalisme n'est pas en réalité l'envers d'un internalisme oublieux de soi, le dehors un dedans retourné comme un gant. 